\title{
Pattern Recognition Based on YIQ Colour Space with Simulated Annealing Algorithm and Optoelectronic Joint Transform Correlation
}

\author{
Chulung Chen, Kaining Gu, Chungcheng Lee, Jianshuen Fang and Yachu Hsieh \\ Department of Photonics Engineering, Yuan Ze University, Taiwan; \\ chulung@saturn.yzu.edu.tw
}

\begin{abstract}
For pattern recognition on various views of the interested colour object, we adopt the YIQ colour space when using simulated annealing algorithm to design the template matching function. Joint transform correlation is devoted for recognition of colour targets. Quantized reference functions are designed for the purpose of display on liquid crystal spatial light modulators. Each reference function is trained with true class images rotated in-plane at 2 degrees intervals between -14 degrees and 14 degrees. Numerical result shows that, generally, YIQ space outperforms conventional RGB space.
\end{abstract}

Keywords: Simulated annealing; Joint transform correlation; Colour pattern recognition; YIQ colour space.

\section{Introduction}

There are two main families of optical correlator, VanderLugt correlator (VLC) [1] and the joint transform correlator (JTC) [2]. VLC was proposed for comparing two signals by utilising the Fourier transforming properties of a lens. In 1966, Weaver and Goodman introduced the JTC for pattern recognition application. A few years later, LCD based JTC [3] proposed by Yu and Lu became an attractive tool for pattern recognition. Since then, the JTC configuration has received increased attention in the past several years because of the less restrictive alignment requirement in comparison with the VLC. However, the classical JTC suffers from strong zero order term (also called DC term) and broad correlation width. The DC term is the sum of each auto-correlation of the reference image and the target image at the output of correlation plane. The existence of the DC term will influence the performance, therefore the removal of the nonzero-order term is of great importance.

To deal with the DC term, Lu et al. [4] adoped phase-shifting technique to design a nonzero- order JTC (NOJTC) and Li et al. [6] used the joint transform power spectrum (JTPS) subtraction strategy to realize the NOJTC. The Mach-Zehnder JTC (MZJTC) [6] can remove the zero-order term in only one step directly without storing the Fourier spectra of both the reference and target images beforehand. Later, Chen et al. $[7,8]$ adopted constraint optimization based on Lagrangian method to yield a sharp correlation peak.

To write reference function and the input test scene onto a spatial light modulator, quantized versions are necessary. On the other hand, the simulated annealing (SA) method $[9,10]$ have been successfully applied to optimization problems. Annealing is a physical process of decreasing temperature gradually in order to reach the global minimum energy states. SA is an effective and commonly numerical optimization algorithm used to solve non linear optimization problems. SA is a Monte Carlo approach 
Chulung Chen, Kaining Gu, Chungcheng Lee, Jianshuen Fang and Yachu Hsieh; Pattern Recognition Based on YIQ Colour Space with Simulated Annealing Algorithm and Optoelectronic J oint Transform Correlation. Advances in I mage and Video Processing, Volume 4 No 5, October (2016); pp: 17-22

to minimize multivariate functions. We will take advantage of this feature for colour pattern recognition.

\section{Analysis}

RGB color model has been widely used and is easy to understand. It consists of the red, green and blue respectively. However, RGB color model is not the most suitable color model on many applications. In this paper, the color seperation to design the reference function (or template) is based on YIQ color space. Y means luminance that represents the achromatic (black and white) image without any color. I and $Q$ are the two chrominance components. I is deviations from orange-luminance to cyan-luminance and $Q$ is deviations from purple-luminance to chartreuse-luminance. It transform RGB source into one luminance and two chrominance components. On the otherhand, the optoelectronic system is based on a MZJTC structure, as shown in Figure. 1. It is consisted of one laser, one spatial filter, one collimated lens (CL), 3 beam splitters (BS), 3 polarizing beam splitters (PBS), 3 Fourier lenses (FL), 3 reflective liquid spatial light modulators (RLCSLM), 3 charge coupled device (CCD) cameras, 1 electronic subtractor (ES) which is used for removing the zero-order term at the final output, and 1 computer for controlling the whole system. Besides, there are 1 half wave plate (HWP) and 1 quarter wave plate (QWP) in front of each RLCSLM. The MZJTC structure is based on the Mach-Zehnder interferometer technique with Stokes relationships. The difference between conventional NOJTC and MZJTC is that the MZJTC structure needs only one step to remove the zero-order term. The processes are presented as follows.

First, 3 colour components of the test colour image are jointly displayed in grayscale at the RLCSLM1. Similarly, 3 colour component of the test colour image are also displayed in grayscale at the RLCSLM2. The target on the RLCSLM1 is illuminated and Fourier optically transformed by FL1. After passing through the PBS3, the irradiance of transmitted and reflected Fourier spectrum is respectively detected by CCD1 and CCD2 in the Fourier frequency domain. Then, the difference of joint Fourier power spectrum between CCD1 and CCD2 is displayed at the RLCSLM3, such that the zero-order term will be subsequently removed at the output. Finally, CCD3 captures another Fouier transform spectrum of the difference. The output contains the overlapping of each cross-correlation of the reference component and the target component. More detailed analysis of MZJTC can be found in the literature [10-12].

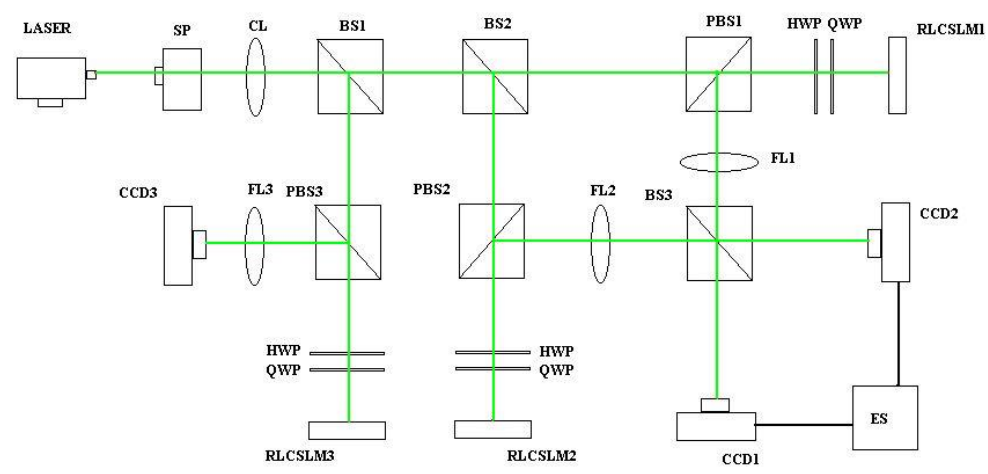

Figure 1. Mach-Zehnder joint transform correlator.

To evaluate the recognition capability, some measurement criteria [13] including correlation peak intensity (CPI) and peak to sidelobe ratio (PSR) are utilized. CPI is the cross-correlation peak intensity 
at the correlation output plane. PSR is the primary correlation peak energy versus secodary peak energy in the region of interest.

\section{Proposed Algorithm}

One colourful insect is selected as the basic pattern of the target, whose size is of $64 \times 64 \times 3$ pixels. It is separated into $Y, I$ and $Q$ channels. For the sake of comparison, another insect is selected as the nontarget. These two images are shown in Figure 2. For simplicity, We rotate these 2 objects in plane from $-14^{\circ}$ to $14^{\circ}$, and select patterns $2^{\circ}$ apart. Totally there are 15 rotationally distorted patterns per object used as the training set for each colour channel. Next, for each training set, we utilize SA algorithm to obtain the reference template. In our study, the CPE (correlation plane energy) is proposed to construct the energy function.
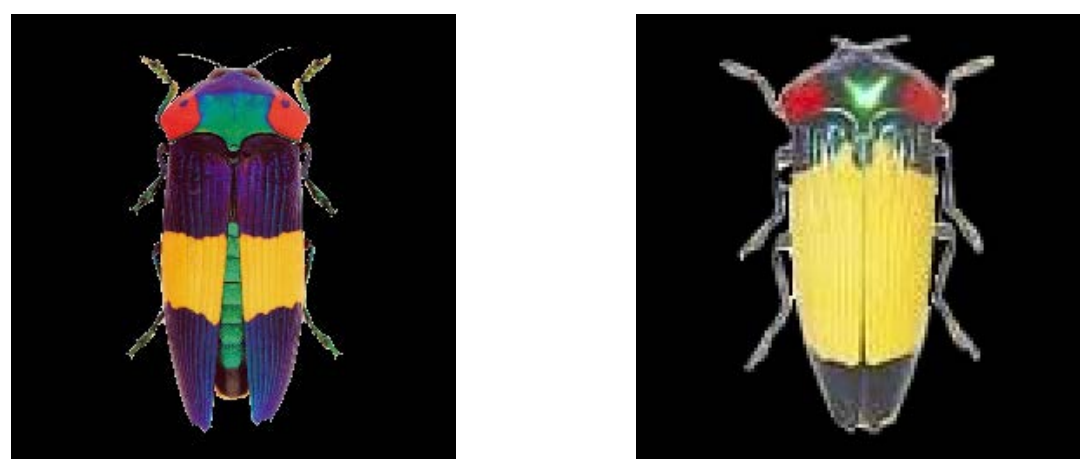

Figure 2: Target (left) and nontarget (right)

SA simulates the cooling process by slowly lowering the system temperature until it converges to a steady, frozen state. The steps of SA algorithm for each channel are similar to those described in our previous paper[14].

- Step 1: Yield the initial reference function randomly.

- Step 2: Calculate CPE and CPI for each training image, then compute the ratio, and add all the ratios together as the energy function $E_{\text {old. }}$. It is expressed as.

$$
E_{\text {old }}=\sum_{i=1}^{N} \frac{\mathrm{CPE}_{\mathrm{i}}}{\mathrm{CPI}_{\mathrm{i}}}
$$

Here $i$ is the index of the training image.

- Step 3: Alter the level number just for one pixel of the reference function $h(x, y)$, and then calculate the new energy function $E_{\text {new. }}$.

- Step 4: If the minimum peak value of the new cross-correlation energy function over all training images is not higher than, say, 0.85 times of the minimum peak value of the old crosscorrelation energy function, the change of the pixel value won't be accepted and the process returns to the step 3.

- Step 5: Calculate the difference of energy functions, which is $\Delta E$ and expressed as

$$
\Delta E=E_{\text {new }}-E_{\text {old }}
$$

- Step 6: If $\Delta E \leq 0$, accept the level number in the new reference function $\mathrm{h}(\mathrm{x}, \mathrm{y})$, set $E_{\text {new }}$ as the next system temperature $\mathrm{T}$, which is the new starting point $E_{\text {old }}$ 
Chulung Chen, Kaining Gu, Chungcheng Lee, Jianshuen Fang and Yachu Hsieh; Pattern Recognition Based on YIQ Colour Space with Simulated Annealing Algorithm and Optoelectronic J oint Transform Correlation. Advances in Image and Video Processing, Volume 4 No 5, October (2016); pp: 17-22

- Step 7: If not, compute the probability. If it is greater than a randomly generated number within the range between 0 and 1 , and then accept the alteration of the pixel value.

- Step 8: Check whether all pixels have been scanned. If they have, move to the next step. Otherwise go back to step 4.

- Step 9: Record the value of energy function in each cycle. If the normalized standard deviation of energy for the last, say, 5 cycles is smaller than, say, 0.03 , and then terminate the computaion and exit the algorithm. Otherwise reduce system temperature typically by, say, $10 \%$, and go back to step 3 .
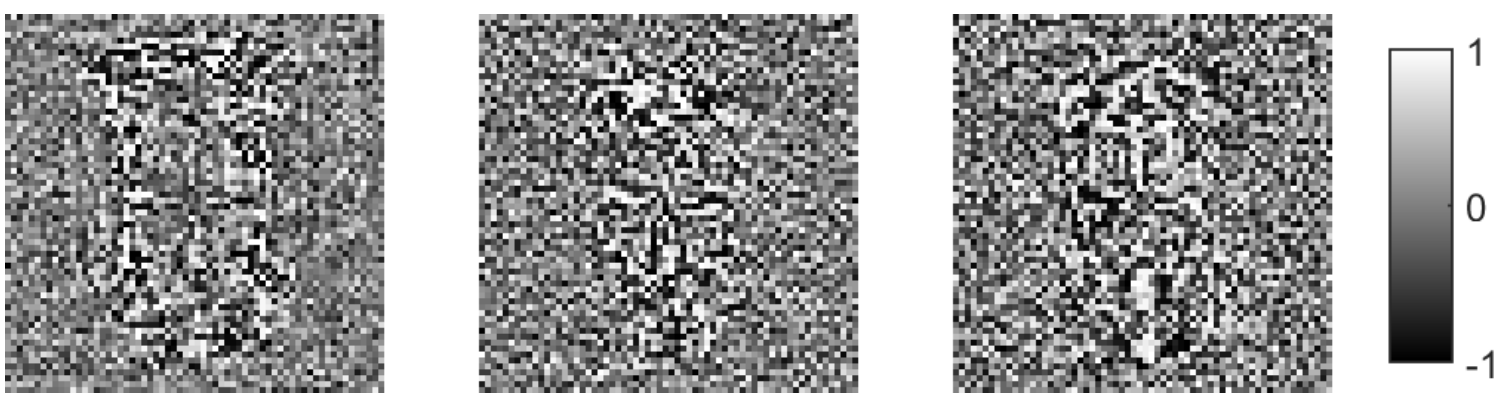

Figure 3: Y, I, Q reference functions calculated by SA algorithm

\section{Result}

Figure 3 shows respectively the synthesized reference function of $\mathrm{Y}, \mathrm{I}, \mathrm{Q}$ components from left to right, in grayscale of 31 levels using SA algorithm. To meet the optical requirement, the value of the grayscale is confined between -1 to 1 , as illustrated by the dynamic range on the right-hand side of the figure. It is worth noting that the CPI is unlikely to be the same for all training targets. Specifically, however, in our proposed technique, the minimum value of the CPI from these training targets is set as the threshold. Furthermore, the correlation intensity has been normalized to a range between 0 and 1 , based on the threshold value. Therefore, values above the threshold CPI are set to 1 . The CPI curve versus the rotation angle for the target as well as for the nontarget are shown in Figure 4 for the purpose of comparison. These 2 curves are seperated considerably. To determine whether the object under test is the target, we can set a threshold value of correlation peak, above which the input can be treated as a target and below which it is a non-target. Figure 5 shows the intensity distribution of the correlation output in the region of interest, where addition of desired cross correlations between the reference and the colour component from all 3 channels occurs. Both the target and nontarget are $0^{\circ}$ rotated. As expected, high correlation peak corresponds to the correct pattern, whereas low correlation profile is observed for the nontarget. We obtain recognition of target and discrimination of nontarget. To see how much YIQ space improves, PSR curve for RGB colour space is also plotted in figure 6 . The curve is lower than that for YIQ curve at each rotation angle of the target. The reason is that, in most cases, when compared with RGB components, YIQ components are less correlated with other. This explains why the correlation profile is sharper for YIQ colour space. 


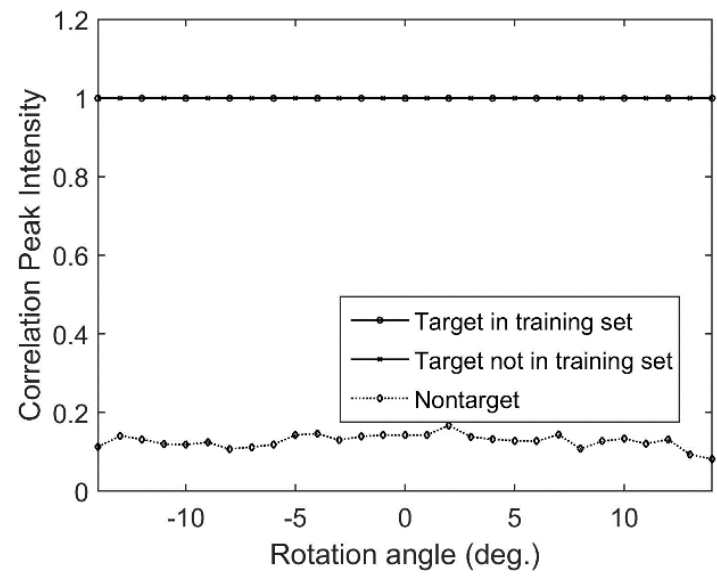

Figure 4: CPI versus rotation angle
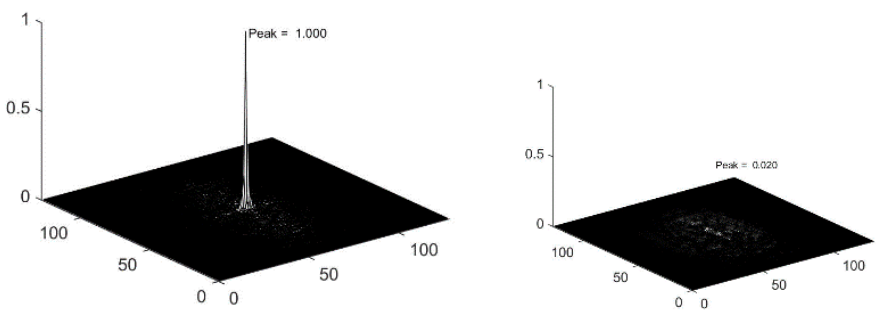

Figure 5: Example of correlation output for target (left) and nontarget (right) without rotation.

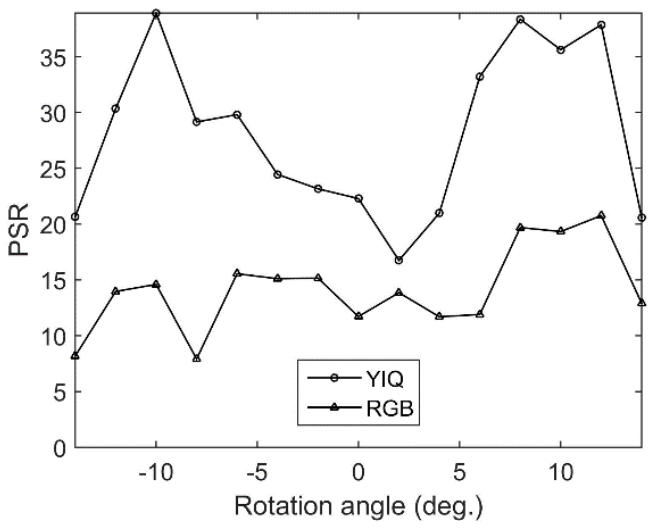

Figure 6: Comparison of the PSR between YIQ and RGB colour spaces as the target rotates.

\section{Conclusion}

In this paper, we have proposed to utilized YIQ colour space together with SA for pattern recognition on JTC. Comparison between YIQ and RGB colour spaces has been evaluated in terms of PSR. The improvement is remarkable. The result verifies the feasibility of our proposed method. It is exactly what we expected to see. The performance for the optoelectronic pattern recognition is promising. In the future work, we will further improve the algorithm.

\section{REFERENCES}

[1]. VanderLugt, A., Signal detection by complex spatial filtering. Information Theory, IEEE Transactions on, 1964. 10(2): p. 139-145. 
Chulung Chen, Kaining Gu, Chungcheng Lee, Jianshuen Fang and Yachu Hsieh; Pattern Recognition Based on YIQ Colour Space with Simulated Annealing Algorithm and Optoelectronic J oint Transform Correlation. Advances in I mage and Video Processing, Volume 4 No 5, October (2016); pp: 17-22

[2]. Weaver, C. S. and J. W. Goodman, A technique for optically convolving two functions. Applied Optics, 1966. 5: p. 1248-1249.

[3]. Yu, F. T. S. and X. J. Lu, A real-time programmable joint-transform correlator. Optics Communications, 1984. 52: p. 10-16

[4]. Lu, G., et al., Imentation of a non-zero-order joint-transform correlator by use of phase-shifting techniques. Applied Optics, 1997. 36: p. 470-483.

[5]. Li, C., S. Yin and F. T. S. Yu, Nonzero-order joint transform correlator. Optical Engineering, 1998. 37: p. 5865.

[6]. Cheng, C. and H. Tu, Implementation of a nonzero-order joint transform correlator using interferometric technique. Optical Review, 2002. 9: p. 193-196.

[7]. Wu, C., C. Chen, and J. Fang, Linearly constrained color pattern recognition with a non-zero order joint transform correlator. Optics Communications, 2002. 214: p. 65-75.

[8]. Chen C., and J. Fang, Optimal synthesis of a real-valued template for synthetic aperture radar pattern recognition. Microwave and Optical Technology Letters, 2002. 32(2): p. 91-95.

[9]. Kirkpatrick, S., et al., Optimization by simulated annealing. Science, 1983. 220: p. 671-680,

[10]. Chen, C. and C. Chen, A Mach-Zehnder joint transform correlator with the simulated annealing algorithm for pattern recognition. Optics Communications, 2011. 284: p. 3946-3953.

[11]. Fu, S., et al., Application of simulated annealing for color pattern recognition to the optoelectronic correlator with liquid crystal device. The 2012 IAENG International Conference on Imaging Engineering. p.683-688.

[12]. Liu, C., et al., Pattern recognition by Mach-Zehnder joint transform correlator with binary power spectrum," Proceedings SPIE 8559.

[13]. Kumar, B. V. K. V. and L. Hassebrook, Performance measures for correlation filters. Applied Optics, 1990. 29: p. 2997-3006.

[14]. Chen, C., et al., Application of simulated annealing for color pattern recognition to hybrid optoelectronic joint transform correlator. Advances in Image and Video Processing, 2015. 3(6): p. 13-17. 